PROCEEDINGS OF THE

AMERICAN MATHEMATICAL SOCIETY

Volume 139, Number 8, August 2011, Pages 2705-271

S 0002-9939(2011)10808-6

Article electronically published on January 14, 2011

\title{
INVARIANT DISTRIBUTIONS ON PROJECTIVE SPACES OVER LOCAL FIELDS
}

\author{
GUYAN ROBERTSON \\ (Communicated by Alexander N. Dranishnikov)
}

\begin{abstract}
Let $\Gamma$ be an $\widetilde{A}_{n}$ subgroup of $\mathrm{PGL}_{n+1}(\mathbb{K})$, with $n \geq 2$, where $\mathbb{K}$ is a local field with residue field of order $q$ and let $\mathbb{P}_{\mathbb{K}}^{n}$ be projective $n$-space over $\mathbb{K}$. The module of coinvariants $H_{0}\left(\Gamma ; C\left(\mathbb{P}_{\mathbb{K}}^{n}, \mathbb{Z}\right)\right)$ is shown to be finite. Consequently there is no nonzero $\Gamma$-invariant $\mathbb{Z}$-valued distribution on $\mathbb{P}_{\mathbb{K}}^{n}$.
\end{abstract}

\section{INTRODUCTION}

Let $\mathbb{K}$ be a nonarchimedean local field with residue field $k$ of order $q$ and uniformizer $\pi$. Denote by $\mathbb{P}_{\mathbb{K}}^{n}$ the set of one-dimensional subspaces of the vector space $\mathbb{K}^{n+1}$, i.e. the set of points in projective $n$-space over $\mathbb{K}$. Then $\mathbb{P}_{\mathbb{K}}^{n}$ is a compact totally disconnected space with the quotient topology inherited from $\mathbb{K}^{n+1}$, and there is a continuous action of $G=\mathrm{PGL}_{n+1}(\mathbb{K})$ on $\mathbb{P}_{\mathbb{K}}^{n}$.

Let $\Gamma$ be a lattice subgroup of $G$. The abelian group $C\left(\mathbb{P}_{\mathbb{K}}^{n}, \mathbb{Z}\right)$ of continuous integer-valued functions on $\mathbb{P}_{\mathbb{K}}^{n}$ has the structure of a $\Gamma$-module, and the module of coinvariants $C\left(\mathbb{P}_{\mathbb{K}}^{n}, \mathbb{Z}\right)_{\Gamma}=H_{0}\left(\Gamma ; C\left(\mathbb{P}_{\mathbb{K}}^{n}, \mathbb{Z}\right)\right)$ is a finitely generated group. Now suppose that $\Gamma$ is an $\widetilde{A}_{n}$ group [3, 4, i.e. $\Gamma$ acts freely and transitively on the vertex set of the Bruhat-Tits building of $G$, which has type $\widetilde{A}_{n}$. A free group is an $\widetilde{A}_{1}$ group since it acts freely and transitively on the vertex set of a tree, which is a building of type $\widetilde{A}_{1}$. For $n \geq 2$, the $\widetilde{A}_{n}$ groups are unlike free groups. This article proves the following.

Theorem 1.1. If $\Gamma$ is an $\widetilde{A}_{n}$ subgroup of $\mathrm{PGL}_{n+1}(\mathbb{K})$, where $n \geq 2$, then $C\left(\mathbb{P}_{\mathbb{K}}^{n}, \mathbb{Z}\right)_{\Gamma}$ is a finite group.

The proof depends upon the fact that $\Gamma$ has Kazhdan's property (T). A distribution on $\mathbb{P}_{\mathbb{K}}^{n}$ is a finitely additive $\mathbb{Z}$-valued measure $\mu$ defined on the clopen subsets of $\mathbb{P}_{\mathbb{K}}^{n}$.

Corollary 1.2. If $\Gamma$ is an $\widetilde{A}_{n}$ subgroup of $\mathrm{PGL}_{n+1}(\mathbb{K})$, where $n \geq 2$, then there is no nonzero $\Gamma$-invariant $\mathbb{Z}$-valued distribution on $\mathbb{P}_{\mathbb{K}}^{n}$.

This contrasts strongly with the main result of $[\underline{8}$ concerning boundary distributions associated with finite graphs. A torsion free lattice subgroup $\Gamma$ of $\mathrm{PGL}_{2}(\mathbb{K})$ is a free group, of rank $r$ say. It was shown in 8 that in this case the group of

Received by the editors August 3, 2010.

2010 Mathematics Subject Classification. Primary 20F65, 20G25, 51E24.

Key words and phrases. Buildings, boundary distributions.

(C)2011 American Mathematical Society Reverts to public domain 28 years from publication 
$\Gamma$-invariant $\mathbb{Z}$-valued distributions on $\mathbb{P}_{\mathbb{K}}^{1}$ is isomorphic to $\mathbb{Z}^{r}$. In particular, there are many such distributions.

\section{BACKGROUND}

2.1. The Bruhat-Tits building. If $\mathbb{K}$ is a local field with discrete valuation $v$ : $\mathbb{K}^{\times} \rightarrow \mathbb{Z}$, let $\mathcal{O}=\{x \in \mathbb{K}: v(x) \geq 0\}$ and let $\pi \in \mathbb{K}$ satisfy $v(\pi)=1$. A lattice $L$ is an $\mathcal{O}$-submodule of $\mathbb{K}^{n+1}$ of rank $n+1$. In other words $L=\mathcal{O} e_{1}+\mathcal{O} e_{2}+\cdots+\mathcal{O} e_{n+1}$ for some basis $\left\{e_{1}, e_{2}, \ldots, e_{n+1}\right\}$ of $\mathbb{K}^{n+1}$. Two lattices $L_{1}$ and $L_{2}$ are equivalent if $L_{1}=\alpha L_{2}$ for some $\alpha \in \mathbb{K}^{\times}$. The Bruhat-Tits building of $\mathrm{PGL}_{n+1}(\mathbb{K})$ is a twodimensional simplicial complex $\Delta$ whose vertices are equivalence classes of lattices in $\mathbb{K}^{n+1}$ 9]. Two lattice classes $\left[L_{0}\right],\left[L_{1}\right]$ are adjacent if, for suitable representatives $L_{1}, L_{2}$, we have $L_{0} \subset L_{1} \subset \pi^{-1} L_{0}$. A simplex is a set of pairwise adjacent lattice classes. The maximal simplices (chambers) are the sets $\left\{\left[L_{0}\right],\left[L_{1}\right], \ldots,\left[L_{n}\right]\right\}$ where $L_{0} \subset L_{1} \subset \cdots \subset L_{n} \subset \pi^{-1} L_{0}$. These inclusions determine a canonical ordering of the vertices in a chamber, up to cyclic permutation. Each vertex $v$ of $\Delta$ has a type $\tau(v) \in \mathbb{Z} /(n+1) \mathbb{Z}$, and each chamber of $\Delta$ has exactly one vertex of each type. If the Haar measure on $\mathbb{K}^{n+1}$ is normalized so that $\mathcal{O}^{n+1}$ has measure 1 , then the type map may be defined by $\tau([L])=\log _{q}(\operatorname{vol}(L))+(n+1) \mathbb{Z}$. The cyclic ordering of the vertices of a chamber coincides with the natural ordering given by the vertex types (Figure 1). Let $E^{1}$ denote the set of directed edges $e=(x, y)$ of $\Delta$ such that $\tau(y)=\tau(x)+1$. Write $o(e)=x$ and $t(e)=y$. The subgraph of the 1-skeleton of $\Delta$ with edge set $E^{1}$ is studied in [5, 7].

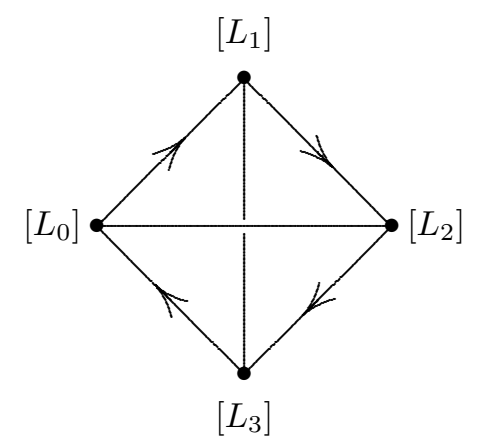

FIgURE 1. $\widetilde{A}_{3}$ case: Cyclic ordering of the vertices of a chamber

Lemma 2.1. Let $C$ be a chamber of $\Delta$. Then $C$ contains $n+1$ directed edges $e \in E^{1}$.

Proof. By [9, Chapter 9.2], there is a basis $\left(e_{1}, \ldots, e_{n+1}\right)$ of $\mathbb{K}^{n+1}$ such that the vertices of $C$ are the classes of the lattices

$$
\begin{aligned}
& L_{0}=\pi \mathcal{O} e_{1}+\pi \mathcal{O} e_{2}+\pi \mathcal{O} e_{3}+\cdots+\pi \mathcal{O} e_{n+1}, \\
& L_{1}=\mathcal{O} e_{1}+\pi \mathcal{O} e_{2}+\pi \mathcal{O} e_{3}+\cdots+\pi \mathcal{O} e_{n+1} \\
& L_{2}=\mathcal{O} e_{1}+\mathcal{O} e_{2}+\pi \mathcal{O} e_{3}+\cdots+\pi \mathcal{O} e_{n+1} \\
& \ldots \ldots \\
& L_{n}=\mathcal{O} e_{1}+\mathcal{O} e_{2}+\mathcal{O} e_{3}+\cdots+\pi \mathcal{O} e_{n+1}
\end{aligned}
$$


Define $L_{n+1}=L_{0}$. Then the edges $C$ which lie in $E^{1}$ are $\left(\left[L_{k}\right],\left[L_{k+1}\right]\right)$, where $0 \leq k \leq n$.

The building $\Delta$ is of type $\widetilde{A}_{n}$, and the action of $\mathrm{GL}_{n+1}(\mathbb{K})$ on the set of lattices induces an action of $\mathrm{PGL}_{n+1}(\mathbb{K})$ on $\Delta$ which is transitive on the vertex set. The action of $\mathrm{PGL}_{n+1}(\mathbb{K})$ on $\Delta$ is type rotating in the sense that, for each $g \in \mathrm{PGL}_{n+1}(\mathbb{K})$, there exists $i \in \mathbb{Z} /(n+1) \mathbb{Z}$ such that $\tau(g v)=\tau(v)+i$ for all vertices $v \in \Delta$.

Fix a vertex $v_{0} \in \Delta$ of type 0 , and let $\Pi\left(v_{0}\right)$ be the set of vertices adjacent to $v_{0}$. Then $\Pi\left(v_{0}\right)$ has a natural incidence structure: if $u, v \in \Pi\left(v_{0}\right)$ are distinct, then $u$ and $v$ are incident if $u, v$ and $v_{0}$ lie in a common chamber of $\Delta$. If $v_{0}$ is the lattice class $\left[L_{0}\right]$, then $\Pi\left(v_{0}\right)$ consists of the classes $[L]$ where $L_{0} \subset L \subset \pi^{-1} L_{0}$, and one can associate to $[L] \in \Pi\left(v_{0}\right)$ the subspace $v=L / L_{0}$ of $\pi^{-1} L_{0} / L_{0} \cong k^{n+1}$. Thus we may identify $\Pi\left(v_{0}\right)$ with the flag complex of subspaces of the vector space $k^{n+1}$. Under this identification, a vertex $v \in \Pi\left(v_{0}\right)$ has type $\tau(v)=\operatorname{dim}(v)+\mathbb{Z} /(n+1) \mathbb{Z}$ where $\operatorname{dim}(v)$ is the dimension of $v$ over $k$. A chamber $C$ of $\Delta$ which contains $v_{0}$ has vertices $v_{0}, v_{1}, \ldots, v_{n}$, where $(0)=v_{0} \subset v_{1} \subset \cdots \subset v_{n} \subset k^{n+1}$ is a complete flag. For brevity, write $C=\left\{v_{0} \subset v_{1} \subset \cdots \subset v_{n}\right\}$.

Definition 2.2. If $e=\left(\left[L_{0}\right],\left[L_{1}\right]\right) \in E^{1}$, where $L_{0} \subset L_{1} \subset \pi^{-1} L_{0}$ and $\tau\left(\left[L_{1}\right]\right)=$ $\tau\left(\left[L_{0}\right]\right)+1$, then define $\Omega(e)$ to be the set of lines $\ell \in \mathbb{P}_{\mathbb{K}}^{n}$ such that $L_{1}=L_{0}+$ $\left(\ell \cap \pi^{-1} L_{0}\right)$. The sets $\Omega(e), e \in E^{1}$, form a basis for the topology on $P_{\mathbb{K}}^{n}$ (cf. [10, Ch. II.1.1], [1, 1.6]).

Lemma 2.3. If $e \in E^{1}$, then $\Omega(e)$ may be expressed as a disjoint union of $q^{n}$ sets

$$
\Omega(e)=\bigsqcup_{\substack{o\left(e^{\prime}\right)=t(e) \\ \Omega\left(e^{\prime}\right) \subset \Omega(e)}} \Omega\left(e^{\prime}\right) .
$$

Proof. Let $e=\left(\left[L_{0}\right],\left[L_{1}\right]\right) \in E^{1}$, where $L_{0} \subset L_{1} \subset \pi^{-1} L_{0}$ and $\tau\left(\left[L_{1}\right]\right)=\tau\left(\left[L_{0}\right]\right)+1$. If $\ell \in \Omega(e)$, then $L_{1}=L_{0}+\left(\ell \cap \pi^{-1} L_{0}\right)$. Choose $e^{\prime}=\left(\left[L_{1}\right],\left[L_{2}\right]\right)$, where $L_{2}=$ $L_{0}+\left(\ell \cap \pi^{-2} L_{0}\right)$. Now $L_{0} \subset L_{1} \subset L_{2} \subset \pi^{-1} L_{1}$ and $L_{2} / L_{1}$ is a 1-dimensional subspace of $\pi^{-1} L_{1} / L_{1} \cong k^{n+1}$. Moreover, $L_{2} / L_{1}$ is not incident with the $n$ dimensional subspace $\pi^{-1} L_{0} / L_{1}$ of $\pi^{-1} L_{1} / L_{1} \cong k^{n+1}$. There are precisely $q^{n}$ such 1-dimensional subspaces of $k^{n+1}$, each of which corresponds to an edge $e^{\prime} \in E^{1}$.

Lemma 2.4. If $\xi$ is a fixed vertex of $\Delta$, then $\mathbb{P}_{\mathbb{K}}^{n}$ may be expressed as a disjoint union

$$
\mathbb{P}_{\mathbb{K}}^{n}=\bigsqcup_{o(e)=\xi} \Omega(e) .
$$

Proof. Let $\xi=\left[L_{0}\right]$, where $L_{0}$ is a lattice. If $\ell \in \mathbb{P}_{\mathbb{K}}^{n}$, define the lattice $L_{1}=$ $L_{0}+\left(\ell \cap \pi^{-1} L_{0}\right)$. Then $L_{0} \subset L_{1} \subset \pi^{-1} L_{0}$ and $\tau\left(\left[L_{1}\right]\right)=\tau\left(\left[L_{0}\right]\right)+1$, since $L_{0}$ is maximal in $L_{1}$. Thus the edge $e=\left(\left[L_{0}\right],\left[L_{1}\right]\right)$ lies in $E^{1}$, and $\ell \in \Omega(e)$.

Lemma 2.5. Let $C$ be a chamber of $\Delta$ and denote the directed edges of $C \cap E^{1}$ by $e_{0}, e_{1}, \ldots, e_{n}$. Then $\mathbb{P}_{\mathbb{K}}^{n}$ may be expressed as a disjoint union

$$
\mathbb{P}_{\mathbb{K}}^{n}=\bigsqcup_{i=0}^{n} \Omega\left(e_{i}\right)
$$


Proof. Let $C$ have a vertex set $\left\{\left[L_{0}\right],\left[L_{1}\right], \ldots,\left[L_{n}\right]\right\}$, where $L_{0} \subset L_{1} \subset \cdots \subset L_{n} \subset$ $\pi^{-1} L_{0}$. Let $\ell=\mathbb{K} a \in \mathbb{P}_{\mathbb{K}}^{n}$, where $a \in \mathbb{K}^{n+1}$ is scaled so that $a \in \pi^{-1} L_{0}-L_{0}$. Then $a \in L_{i+1}-L_{i}$ for some $i$, where $L_{i+1} / L_{i} \cong k$ and $L_{n+1}=\pi^{-1} L_{0}$. Thus $\ell \in \Omega\left(e_{i}\right)$.

2.2. $\widetilde{A}_{n}$ groups. From now on let $\Pi=\Pi\left(v_{0}\right)$, the set of neighbours of the fixed vertex $v_{0} \in \Delta$. Thus $\Pi$ is isomorphic to the flag complex of subspaces of $k^{n+1}$, and a chamber $C$ of $\Delta$ which contains $v_{0}$ is a complete flag $\left\{v_{0} \subset v_{1} \subset \cdots \subset v_{n}\right\}$. For $1 \leq r \leq n$, let $\Pi_{r}=\left\{u \in \Pi\left(v_{0}\right): \operatorname{dim} u=r\right\}$.

Now suppose that $\Gamma$ is an $\widetilde{A}_{n}$ group, i.e. $\Gamma$ acts freely and transitively on the vertex set of $\Delta\left[3\right.$, 4]. Then for each $v \in \Pi\left(v_{0}\right)$, there is a unique element $g_{v} \in \Gamma$ such that $g_{v} v_{0}=v$. If $v \in \Pi\left(v_{0}\right)$, then $g_{v}^{-1} v_{0}$ also lies in $\Pi\left(v_{0}\right)$, and $\lambda(v)=g_{v}^{-1} v_{0}$ defines an involution $\lambda: \Pi\left(v_{0}\right) \rightarrow \Pi\left(v_{0}\right)$ such that $g_{\lambda(v)}=g_{v}^{-1}$. Let $\mathcal{T}=\{(u, v, w) \in$ $\left.\Pi\left(v_{0}\right)^{3}: g_{u} g_{v} g_{w}=1\right\}$. If $(u, v, w) \in \mathcal{T}$, then $w$ is uniquely determined by $(u, v)$ and there is a bijective correspondence between triples $(u, v, w) \in \mathcal{T}$ and directed triangles $\left(v_{0}, \lambda(u), v\right)$ of $\Delta$ containing $v_{0}$. By [ 6 , Proposition 2.2], the abstract group $\Gamma$ has a presentation with generating set $\left\{g_{v}: v \in \Pi\left(v_{0}\right)\right\}$ and relations

$$
\begin{aligned}
& g_{u} g_{\lambda(u)}=1, \quad u \in \Pi\left(v_{0}\right), \\
& g_{u} g_{v} g_{w}=1, \quad(u, v, w) \in \mathcal{T} .
\end{aligned}
$$

If $u \in \Pi\left(v_{0}\right)$, then $\tau\left(g_{u} v_{0}\right)=\tau(u)=\tau(u)+\tau\left(v_{0}\right)$. Hence $\tau\left(g_{u} x\right)=\tau(u)+\tau(x)$ for each vertex $x$ of $\Delta$, since $g_{u}$ is type rotating. In particular, if $u, v \in \Pi\left(v_{0}\right)$, then

$$
\tau\left(g_{u} g_{v} v_{0}\right)=\tau(u)+\tau(v) .
$$

It follows from (5) that

$$
\tau(\lambda(u))=-\tau(u)
$$

for each $u \in \Pi$. Also, if $(u, v, w) \in \mathcal{T}$, then

$$
\tau(u)+\tau(v)+\tau(w)=0 .
$$

Let $C=\left\{v_{0} \subset v_{1} \subset \cdots \subset v_{n}\right\}$ be a chamber of $\Delta$ containing $v_{0}$. Since the vertices $v_{i-1}$ and $v_{i}$ are adjacent, so are the vertices $v_{0}=g_{v_{i-1}}^{-1} v_{i-1}$ and $g_{v_{i-1}}^{-1} g_{v_{i}} v_{0}=$ $g_{v_{i-1}}^{-1} v_{i}$. Also, $\tau\left(g_{v_{i-1}}^{-1} g_{v_{i}} v_{0}\right)=\tau\left(v_{i}\right)-\tau\left(v_{i-1}\right)=1$. Therefore $g_{v_{i-1}}^{-1} g_{v_{i}}=g_{a_{i}}$, where $a_{i} \in \Pi_{1}, v_{n+1}=v_{0}$ and $g_{v_{0}}=1$. Thus $g_{a_{1}} g_{a_{2}} \ldots g_{a_{k}}=g_{v_{k}}(1 \leq k \leq n)$ and $g_{a_{1}} g_{a_{2}} \ldots g_{a_{n+1}}=1$.

The $(n+1)$-tuple $\sigma(C)=\left(a_{1}, a_{2}, \ldots, a_{n+1}\right) \in \Pi_{1}^{n+1}$ is uniquely determined by the chamber $C$ containing $v_{0}$. Denote by $\mathfrak{S}$ the set of all $(n+1)$-tuples $\sigma(C)$ associated with such chambers $C$. If $u \in \Pi\left(v_{0}\right)$ with $\operatorname{dim}(u)=k$, then $u$ is a vertex of a chamber $C$ containing $v_{0}$. Therefore

$$
g_{u}=g_{a_{1}} g_{a_{2}} \ldots g_{a_{k}}, \quad \text { where } \quad a_{i} \in \Pi_{1}, 1 \leq i \leq k .
$$

In particular, the set $\left\{g_{a}: a \in \Pi_{1}\right\}$ generates $\Gamma$. Since $g_{\lambda(u)}=g_{u}^{-1}$, we have

$$
g_{\lambda(u)}=g_{a_{i+1}} \ldots g_{a_{n+1}} .
$$

Note that the expression (6) for $g_{u}$ is not unique, but depends on the choice of the chamber $C$ containing $u$ and $v_{0}$. An edge in $E^{1}$ has the form $\left(x, g_{a} x\right)$, where $a \in \Pi_{1}$.

Lemma 2.6. The $\widetilde{A}_{n}$ group $\Gamma$ has a presentation with generating set $\left\{g_{a}: a \in \Pi_{1}\right\}$ and relations

$$
g_{a_{1}} g_{a_{2}} \ldots g_{a_{n+1}}=1, \quad\left(a_{1}, a_{2}, \ldots, a_{n+1}\right) \in \mathfrak{S} .
$$


Proof. It is enough to show that the relations (4) follow from the relations (8). Let $(u, v, w) \in \mathcal{T}$ with $\operatorname{dim}(u)=i, \operatorname{dim} v=j$ and $\operatorname{dim} w=k$, where $i+j+$ $k \equiv 0 \bmod (n+1)$. Choose a chamber $C=\left\{v_{0} \subset v_{1} \subset \cdots \subset v_{n}\right\}$ containing $\left\{v_{0}, g_{u} v_{0}, g_{u} g_{v} v_{0}\right\}$. Let $\left(a_{1}, a_{2}, \ldots, a_{n+1}\right)=\sigma(C) \in \Pi_{1}^{n+1}$ be the element of $\mathfrak{S}$ determined by $C$. Then $g_{u} v_{0}$ is the vertex of $C$ of type $i$, so $g_{u}=g_{a_{1}} g_{a_{2}} \ldots g_{a_{i}}$.

Suppose that $j<n+1-i$. Then $g_{u} g_{v} v_{0}$ is the vertex of $C$ of type $i+j$ and $g_{u} g_{v}=g_{a_{1}} g_{a_{2}} \ldots g_{a_{i+j}}$. Thus $g_{v}=g_{a_{i+1}} \ldots g_{a_{i+j}}$ and $g_{w}=g_{a_{i+j+1}} \ldots g_{a_{n+1}}$. Therefore

$$
g_{u} g_{v} g_{w}=g_{a_{1}} g_{a_{2}} \ldots g_{a_{n+1}} .
$$

Suppose that $j>n+1-i$. Then $g_{u} g_{v} v_{0}$ has type $i+j-n-1$ and

$$
g_{u} g_{v}=g_{a_{1}} g_{a_{2}} \ldots g_{a_{i+j-n-1}}=g_{a_{1}} g_{a_{2}} \ldots g_{a_{n+1}} g_{a_{1}} \ldots g_{a_{i+j-n-1}} .
$$

Thus $g_{v}=g_{a_{i+1}} \ldots g_{a_{n+1}} g_{a_{1}} \ldots g_{a_{i+j-n-1}}$ and $g_{w}=g_{a_{i+j-n}} \ldots g_{a_{n+1}}$. Therefore

$$
g_{u} g_{v} g_{w}=\left(g_{a_{1}} g_{a_{2}} \ldots g_{a_{n+1}}\right)^{2} .
$$

In each case the relations (4b) follow from the relations (8). The same is true for the relations (4a), by equation (7).

\section{The COINVARIANTS}

If $\Gamma$ is an $\widetilde{A}_{n}$ group acting on $\Delta$, then $\Gamma$ acts on $\mathbb{P}_{\mathbb{K}}^{n}$, and the abelian group $C\left(\mathbb{P}_{\mathbb{K}}^{n}, \mathbb{Z}\right)$ has the structure of a $\Gamma$-module, with $(g \cdot f)(\ell)=f\left(g^{-1} \ell\right), g \in \Gamma, \ell \in$ $\mathbb{P}_{\mathbb{K}}^{n}$. The module of coinvariants, $C\left(\mathbb{P}_{\mathbb{K}}^{n}, \mathbb{Z}\right)_{\Gamma}$, is the quotient of $C\left(\mathbb{P}_{\mathbb{K}}^{n}, \mathbb{Z}\right)$ by the submodule generated by $\left\{g \cdot f-f: g \in \Gamma, f \in C\left(\mathbb{P}_{\mathbb{K}}^{n}, \mathbb{Z}\right)\right\}$. If $f \in C\left(\mathbb{P}_{\mathbb{K}}^{n}, \mathbb{Z}\right)$, then let $[f]$ denote its class in $C\left(\mathbb{P}_{\mathbb{K}}^{n}, \mathbb{Z}\right)_{\Gamma}$. Also, let $\mathbf{1}$ denote the constant function defined by $\mathbf{1}(\ell)=1$ for $\ell \in \mathbb{P}_{\mathbb{K}}^{n}$, and let $\varepsilon=[\mathbf{1}]$.

If $e \in E^{1}$, let $\chi_{e}$ be the characteristic function of $\Omega(e)$. For each $g \in \Gamma$, the functions $\chi_{e}$ and $g \cdot \chi_{e}=\chi_{g e}$ project to the same element in $C\left(\mathbb{P}_{\mathbb{K}}^{n}, \mathbb{Z}\right)_{\Gamma}$. Any edge $e \in E^{1}$ is in the $\Gamma$-orbit of some edge $\left(v_{0}, g_{a} v_{0}\right)$, where $a \in \Pi_{1}$ is uniquely determined by $e$. Therefore it makes sense to denote by $[a]$ the class of $\chi_{e}$ in $C\left(\mathbb{P}_{\mathbb{K}}^{n}, \mathbb{Z}\right)_{\Gamma}$.

Lemma 3.1. The group $C\left(\mathbb{P}_{\mathbb{K}}^{n}, \mathbb{Z}\right)_{\Gamma}$ is finitely generated, with generating set $\{[a]$ : $\left.a \in \Pi_{1}\right\}$.

Proof. Every clopen set $V$ in $\mathbb{P}_{\mathbb{K}}^{n}$ may be expressed as a finite disjoint union of sets of the form $\Omega(e), e \in E^{1}$. Any function $f \in C\left(\mathbb{P}_{\mathbb{K}}^{n}, \mathbb{Z}\right)$ is bounded, by compactness of $\mathbb{P}_{\mathbb{K}}^{n}$, and so takes finitely many values $n_{i} \in \mathbb{Z}$. Therefore $f$ may be expressed as a finite sum $f=\sum_{j} n_{j} \chi_{e_{j}}$, with $e_{j} \in E^{1}$. The result follows, since $\left\{\left[\chi_{e}\right]: e \in E^{1}\right\}=$ $\left\{[a]: a \in \Pi_{1}\right\}$.

Suppose that $e, e^{\prime} \in E^{1}$ with $o\left(e^{\prime}\right)=t(e)=x$, so that $o(e)=g_{\lambda(a)} x$ and $t\left(e^{\prime}\right)=g_{b} x$ for (unique) $a, b \in \Pi_{1}$. Then, by the proof of Lemma 2.3, $\Omega\left(e^{\prime}\right) \subset \Omega(e)$ if and only if $b \cap \lambda(a)=(0)$.

Equations (11) and (2) imply the following relations in $C\left(\mathbb{P}_{\mathbb{K}}^{n}, \mathbb{Z}\right)_{\Gamma}$ :

$$
\begin{aligned}
\varepsilon & =\sum_{a \in \Pi_{1}}[a], \\
{[a] } & =\sum_{\substack{b \in \Pi_{1} \\
b \cap \lambda(a)=(0)}}[b], \quad a \in \Pi_{1} .
\end{aligned}
$$


It is easy to see that $\left|\Pi_{1}\right|=\frac{q^{n+1}-1}{q-1}$. If $a \in \Pi_{1}$, then $\lambda(a) \in \Pi_{n}$, and so the number of elements $b \in \Pi_{1}$ which are incident with $\lambda(a)$ is $\frac{q^{n}-1}{q-1}$. Thus there exist $q^{n}$ elements $b \in \Pi_{1}$ such that $b \cap \lambda(a)=(0)$. In other words, the right side of $(9 \mathrm{~b})$ contains $q^{n}$ terms. As a first step towards proving that $C\left(\mathbb{P}_{\mathbb{K}}^{n}, \mathbb{Z}\right)_{\Gamma}$ is finite, we show that the element $\varepsilon=[\mathbf{1}]$ has finite order.

Lemma 3.2. In the group $C\left(\mathbb{P}_{\mathbb{K}}^{n}, \mathbb{Z}\right)_{\Gamma},\left(q^{n}-1\right) \varepsilon=0$.

Proof. By (9a) and (9b),

$$
\varepsilon=\sum_{a \in \Pi_{1}}[a]=\sum_{a \in \Pi_{1}}\left(\sum_{\substack{b \in \Pi_{1} \\ b \cap \lambda(a)=(0)}}[b]\right)=\sum_{b \in \Pi_{1}} q^{n}[b]=q^{n} \varepsilon .
$$

We can now prove Theorem 1.1. It follows from (3) that if $\left(a_{1}, a_{2}, \ldots, a_{n+1}\right) \in \mathfrak{S}$, then

$$
\sum_{i=1}^{n+1}\left[a_{i}\right]=\varepsilon
$$

Therefore, by Lemmas 2.6 and 3.1 there is a homomorphism $\theta$ from $\Gamma$ onto the abelian group $C\left(\mathbb{P}_{\mathbb{K}}^{n}, \mathbb{Z}\right)_{\Gamma} /\langle\varepsilon\rangle$ defined by $\theta\left(g_{a}\right)=[a]+\langle\varepsilon\rangle$, for $a \in \Pi_{1}$.

The $\widetilde{A}_{n}$ group $\Gamma$ has Kazhdan's property $(\mathrm{T})[2$, Theorems 1.6.1 and 1.7.1]. It follows that $C\left(\mathbb{P}_{\mathbb{K}}^{n}, \mathbb{Z}\right)_{\Gamma} /\langle\varepsilon\rangle$ is finite [2, Corollary 1.3.5]. Therefore $C\left(\mathbb{P}_{\mathbb{K}}^{n}, \mathbb{Z}\right)_{\Gamma}$ is also finite, since $\langle\varepsilon\rangle$ is finite, by Lemma 3.2 .

Distributions. A distribution on $\mathbb{P}_{\mathbb{K}}^{n}$ is a finitely additive $\mathbb{Z}$-valued measure $\mu$ defined on the clopen subsets of $\mathbb{P}_{\mathbb{K}}^{n}[1,1.4]$. By integration, a distribution may be regarded as a $\mathbb{Z}$-linear function on the group $C\left(\mathbb{P}_{\mathbb{K}}^{n}, \mathbb{Z}\right)$. Therefore a $\Gamma$-invariant distribution defines a homomorphism $C\left(\mathbb{P}_{\mathbb{K}}^{n}, \mathbb{Z}\right)_{\Gamma} \rightarrow \mathbb{Z}$. This homomorphism is necessarily trivial, since $C\left(\mathbb{P}_{\mathbb{K}}^{n}, \mathbb{Z}\right)_{\Gamma}$ is finite. This proves Corollary 1.2

\section{REFERENCES}

[1] G. Alon and E. de Shalit, On the cohomology of Drinfel'd's p-adic symmetric domain, Israel J. Math. 129 (2002), 1-20. MR1910929(2003i:14019)

[2] B. Bekka, P. de la Harpe and A. Valette, Kazhdan's Property (T), Cambridge University Press, Cambridge, 2008. MR2415834 (2009i:22001)

[3] D. I. Cartwright, Groups acting simply transitively on the vertices of a building of type $\tilde{A}_{n}$, Groups of Lie type and their geometries, W. M. Kantor and L. Di Martino, editors, 43-76. Cambridge University Press, 1995. MR1320514 (96a:20039)

[4] D. I. Cartwright and T. Steger, A family of $\bar{A}_{n}$ groups, Israel J. Math. 103 (1998), 125-140. MR.1613560 (99e:20040)

[5] D. I. Cartwright, P. Solé and A. Żuk, Ramanujan geometries of type $\widetilde{A}_{n}$, Discrete Math. 269 (2003), 35-43. MR.1989451(2004e:05113)

[6] D. I. Cartwright, A. M. Mantero, T. Steger and A. Zappa, Groups acting simply transitively on the vertices of a building of type $\widetilde{A}_{2}$. I, Geom. Ded. 47 (1993), 143-166. MR 1232965 (95b:20053)

[7] A. Lubotzky, B. Samuels and U. Vishne, Ramanujan complexes of type $\widetilde{A}_{d}$, Israel J. Math. 149 (2005), 267-299. MR2191217 (2006i:11134) 
[8] G. Robertson, Invariant boundary distributions associated with finite graphs, J. Combin. Theory Ser. A 115 (2008), 1272-1278. MR.2450343 (2009h:05106)

[9] M. Ronan, Lectures on Buildings, University of Chicago Press, 2009. MR 2560094 (2010i:20002)

[10] J.-P. Serre, Trees, Springer-Verlag, Berlin, 1980. MR607504(82c:20083)

School of Mathematics and Statistics, University of Newcastle, Newcastle upon Tyne, NE1 7RU, United Kingdom

E-mail address: a.g.robertson@ncl.ac.uk 